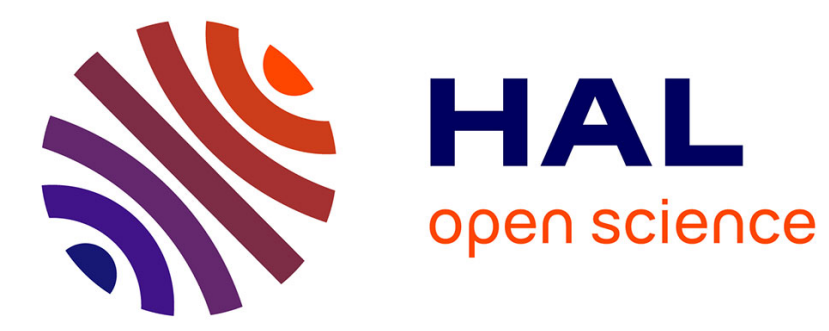

\title{
Receptivity to synthetic jet actuation in boundary layer flows
}

Andrea Palumbo, Onofrio Semeraro, Jean-Christophe Robinet, Luigi de Luca

\section{To cite this version:}

Andrea Palumbo, Onofrio Semeraro, Jean-Christophe Robinet, Luigi de Luca. Receptivity to synthetic jet actuation in boundary layer flows. AIAA Scitech 2020 Forum, Jan 2020, Orlando, FL, United States. 10.2514/6.2020-0099 . hal-03087084

\section{HAL Id: hal-03087084 https://hal.science/hal-03087084}

Submitted on 23 Dec 2020

HAL is a multi-disciplinary open access archive for the deposit and dissemination of scientific research documents, whether they are published or not. The documents may come from teaching and research institutions in France or abroad, or from public or private research centers.
L'archive ouverte pluridisciplinaire HAL, est destinée au dépôt et à la diffusion de documents scientifiques de niveau recherche, publiés ou non, émanant des établissements d'enseignement et de recherche français ou étrangers, des laboratoires publics ou privés. 
See discussions, stats, and author profiles for this publication at: https://www.researchgate.net/publication/338396595

\section{Receptivity to synthetic jet actuation in boundary layer flows}

Conference Paper · January 2020

DOI: $10.2514 / 6.2020-0099$

CITATIONS

4 authors, including:

Q11: Onofrio Semeraro

Computer Sciences Laboratory for Mechanics and Engineering Sciences

31 PUBLICATIONS 481 CITATIONS

SEE PROFILE

Luigi De Luca

University of Naples Federico II

155 PUBLICATIONS 1,452 CITATIONS

SEE PROFILE

Some of the authors of this publication are also working on these related projects:

Project Instabilities and Bifurcations in fluid dynamics View project

Project Receptivity for transitional flows View project
READS

211

Jean-Christophe Robinet

Ecole Nationale Supérieure d'Arts et Métiers

159 PUBLICATIONS 1,590 CITATIONS

SEE PROFILE 


\title{
Receptivity to synthetic jet actuation in boundary layer flows
}

\author{
Andrea Palumbo* \\ Università degli Studi di Napoli “Federico II", Department of Industrial Engineering, \\ P.le Tecchio 80, 80125 Naples, Italy \\ Onofrio Semeraro $^{\dagger}$ \\ Laboratoire d'informatique pour la mécanique et les sciences de l'ingénieur (LIMSI), \\ CNRS, Université Paris-Saclay, Bat. 507, Rue John Von Neumann, 91400 Orsay, France \\ Jean-Christophe Robinet \\ DynFluid Laboratory, Arts et Métiers ParisTech, 151 Bd. de l'Hopital, 75013 Paris, France \\ Luigi de Luca ${ }^{\S}$ \\ Università degli Studi di Napoli "Federico II", Department of Industrial Engineering, \\ P.le Tecchio 80, 80125 Naples, Italy
}

The flow field generated by the interaction between a zero-net-mass-flux (synthetic) jet and a boundary layer crossflow is studied in the present work. The investigation consists in Direct Numerical Simulations (DNS) of the resulting unsteady field, for various boundary layer Reynolds numbers $R e_{\delta_{0}^{*}}^{*}$ and jet reduced frequencies $F^{+}$. In particular, the actuation frequency is chosen in order to match the unstable (or less stable) frequency of the incoming crossflow. The analysis is focused on the near-field behaviour of the flow, i.e. next to the jet exit orifice, and in the downstream region. It has been found that the presence of the jet deeply modifies the boundary layer transition. Indeed, the instantaneous flow field is characterized by the presence of unsteady, hairpin-like vortical structures, similar to the ones generated by localized roughness elements. Moreover, streaky structures can be detected in the time-averaged flow field. The overall behaviour of the flow field (investigated via time-averaged boundary layer integral parameters) allows to state that early transition can be achieved by using SJ devices. This work is part of a wider project which aims at realizing a reduced-order modeling of a piezo-driven synthetic jet in crossflow, based on simulations and lumped element modeling of the actuator.

\section{Nomenclature}

$\begin{array}{ll}N & =\text { order of the velocity field polynomials } \\ L_{x}, L_{y}, L_{z} & =\text { dimensions of the (external) computational domain } \\ D & =\text { pipe diameter } \\ \delta_{0}^{*} & =\text { boundary layer displacement thickness at the nozzle center (without the jet) } \\ x_{0} & =\text { virtual distance of the nozzle center from the leading edge of the plate } \\ R e_{\delta_{0}^{*}} & =\text { boundary layer Reynolds number at the nozzle center position (based on } \delta_{0}^{*} \text { ) } \\ \operatorname{Re}_{x_{0}} & =\text { boundary layer Reynolds number at the nozzle center position (based on } x_{0} \text { ) } \\ W o & =\text { jet Womersley number } \\ \omega & =\text { actuation circular frequency } \\ H & =\text { pipe length } \\ U_{\infty} & =\text { freestream velocity } \\ v & =\text { kinematic viscosity } \\ f & =\text { actuation frequency }\end{array}$

\footnotetext{
*Ph.D. student, palumbo.and@gmail.com (Corresponding Author).

${ }^{\dagger}$ Research scientist.

†Professor.

${ }^{\S}$ Professor, Senior Member AIAA.
} 
$=$ actuation period

$=$ space-averaged jet exit velocity at peak blowing

$=$ jet-to-crossflow velocity ratio

$=$ reduced actuation frequency

$=$ jet Reynolds number (based on the maximum velocity amplitude)

$=$ jet momentum coefficient

$=$ averaged jet velocity

$=$ stroke length

$\begin{array}{ll}R e_{0} & =\text { jet Reynolds number (based on } V_{0} \text { ) } \\ S t_{0} & =\text { jet Strouhal number (based on } V_{0} \text { ) }\end{array}$

$\omega^{*} \quad=$ non-dimensional frequency of the boundary layer based on $U_{\infty} / \delta_{0}^{*}$

$F \quad=$ non-dimensional frequency of the boundary layer based on $U_{\infty}^{2} / v$

$-\alpha_{i} \quad=\quad$ spatial growth rate (from linear stability theory)

$\bar{\delta}^{*} \quad=$ time-averaged boundary layer displacement thickness

$\bar{\theta} \quad=\quad$ time-averaged boundary layer momentum thickness

$\bar{H} \quad=$ time-averaged boundary layer shape factor

$\bar{C}_{f} \quad=$ time-averaged boundary layer skin friction coefficient

$U_{p} \quad=$ time-averaged deviation of the controlled flow from the Blasius profile

\section{Introduction}

great effort has been paid in the last years to reduce the pollutant emissions produced by civil aircrafts. As a general Aissue, the environmental impact of the airplane may be reduced by working on the external aerodynamics of the airplane, its electronic systems, structures and engines. In particular, the research on the external aerodynamics focused mainly on drag reduction techniques, based on laminar wings/fuselages, riblets and active flow control (AFC) systems. AFC techniques are commonly employed to suppress/enhance turbulence and reduce the extent of the separated zones on the lifting surfaces, which are key factors of the vehicle performances. In this framework, synthetic jet (SJ) actuators have been proven to be useful devices, due to their unique ability to transfer momentum to the incoming crossflow with a null-net-mass-flow-rate (per actuation cycle) across the jet boundary. For this reason they do not require any pumping system (namely, they are synthetized within the fluid flow to be controlled), and in many cases they are preferred to other active flow control systems, satisfying also the request of low weight and reduced size.

Several techniques to produce a synthetic jet can be found in literature; however two methods have been extensively studied in the last years, namely the piezo-driven [1] and plasma synthetic jet actuators (PSJ) [2]. In the former one, the jet is created by periodic volume variations of a resonant cavity, produced by the deformation of a piezo-electric element located on the bottom wall of the cavity. In the latter one, a high-speed and high-temperature jet is obtained from an electrical discharge between two or three electrodes, enclosed in a small cavity. The high-pressure plasma formed by the discharge exhausts through an orifice, converting the internal energy of the air into the jet kinetic one. In the end, fresh air is ingested within the cavity, refilling the device for the next cycle. The choice of the actuator to be used for a certain application depends on the desired exit velocity and applied frequency, the available energy and space for the actuator, and safety/reliability considerations.

Many works have been recently devoted to the application of SJ actuators to control aerodynamic flows, both in industrial applications and research projects. The former studies are typically devoted to evaluate the effectiveness of such devices to improve the performances of an aircraft. In this framework, the works are also intended to solve practical problems and give design guidelines for the implementation of the actuators on aircraft components, such as wings [3] and vertical tails [4]. The potential impact of this technology on the performances of the aircraft (for example, in terms of reduced fuel consumption) has been also discussed [5].

The latter studies, instead, generally investigate the interaction between the jet and canonical flows such as flat-plate laminar boundary layers, circular cylinder in crossflow [6], liquid or gas free jets [7, 8]. The main aim of these studies is to identify the vortical structures of the unforced flow field and to understand how they are affected by the actuation of the SJ. The findings are useful to shed light on the flow physics when more complex phenomena are involved. For instance, the application of a SJ in a boundary layer can give information on the control of a lifting surface at low angles of attack, whereas the interaction between a SJ and the wake behind a bluff body can mimic the separation control in stall and post-stall configurations. The analyses have been generally carried out in the frequency domain, often with 
low-order reconstructions of the controlled flow field. It has been found that synthetic jets are able to interact with the crossflow by annihilating or enhancing some modes, or introducing new vortical structures into the flow field.

Among the various crossflows analysed in these investigations, a special attention has been devoted to the case of boundary layer; early works were concerned with continuous jets in crossflow, carried out using experimental measurements, flow visualization techniques, direct or large eddy simulations and stability analyses. Various fluid-dynamics instabilities have been detected using global stability analyses. Indeed, the complex flow field generated by a jet issuing in a boundary layer flow is dominated by the interaction of three common types of instabilities, a shear layer Kelvin-Helmholtz instability in the jet region, a (possible) elliptic instability of the counter-rotating vortex pair, and a vortex shedding mechanism, similar to the von Kármán vortex street behind a cylinder, in the near-wall region [9, 10]. These recent analyses strengthen the findings of earlier experimental works [11, 12], exploring a wider range of velocity ratios (between the jet and the unperturbed boundary layer) and crossflow Reynolds numbers.

On the other hand, a smaller number of works concerned the interaction of synthetic jets with a boundary layer, both experimentally and via simulations. The vortical structures generated by a piezo-driven SJ issuing in a boundary layer have been investigated in detail in [13-15] for laminar boundary layers and in [16-20] for turbulent boundary layers. Similar studies have been carried out also for plasma SJs [21]. Besides, a few studies focused on the desired characteristics to be obtained in the controlled flow field. Synthetic jets were typically employed to obtain the so-called virtual aeroshaping, [22]: SJs can generate time-averaged recirculation regions that are larger than the actuator diameter, which can be used to introduce a "virtual morphing" of the aerodynamic surfaces. This behaviour can be achieved when the actuation frequency is much larger than the characteristic frequency of the uncontrolled flow field (which, nonetheless, is generally an unknown quantity): such a technique has been successfully used to control the flow over airfoils [23], bubbles separation [24], and bluff body wakes.

Notwithstanding all the cited papers, the optimized design of a new actuator for a fixed crossflow condition is still a difficult task, since its optimization involves many geometric and structural characteristics of the actuator. Moreover, the device should be able to control the incoming crossflow in a wide range of crossflow conditions. For these reasons, a huge number of experimental tests or numerical simulations should be planned to properly design an actuator, which should lead to intractable computational and experimental costs. However, reduced-order modeling (ROM) has gained an increasing interest in the last decades, in order to reduce the required resources for the design of the actuator and its implementation [25]. Physical modelling of piezo-driven and plasma synthetic jet actuators, based on lumped approximations of the thermo-fluid-dynamic quantities in the cavity and at the exit orifice, has been originally developed for the case of a single SJ actuator, communicating with the quiescent environment through a single orifice or slot [26-29]. Further developments led to dedicated lumped-element models (LEM) of complex devices, with more cavities and/or orifices [30, 31]. The flow field generated by unconventional SJ actuators, with noncircular orifices or multi-slot and multi-cavity characteristics are still object of current research [32-34], and these studies could be the basis for new LEMs. On the contrary, there are no LEMs for the synthetic jets in crossflow, which led the researchers to rely to empirical models or trial-and-error techniques for the design of the actuators.

The present study approaches the problem of realizing a reduced-order modeling of a piezo-driven synthetic jet in crossflow, based on simulations and lumped element modeling of the actuator. Direct numerical simulations of the flow field generated by the interaction between the synthetic jet and the crossflow have been carried out, in order to recognize the vortical structures arising in the flow field. In the present investigation the actuator is not fully included in the CFD simulations, but it is simply represented by an oscillating inlet condition, enforced at the orifice lower end. However, this approach is suitable for integration in a LEM-based design of the actuator: the jet velocity at the orifice exit is obtained via existing LEM models for the jet in quiescent conditions, and changes in geometry, actuation frequency and supplied voltage can be easily considered with the LEM formulation. This procedure allows also to include the compressibility effects of the flow in the cavity and the coupling mechanisms between the diaphragm motion and the fluid flow (Helmholtz resonance). Differently from the virtual aeroshaping studies, in this case the actuation frequency is next to the characteristic frequency of the crossflow, in order to deepen the understanding of how SJs modify the modes of the uncontrolled flow. The characteristic frequency of the Blasius boundary layer is evaluated via standard linear stability theory.

The paper is organized as follows: the numerical setup (CFD code, numerical domain, boundary conditions, flow parameters and dimensionless quantities) is described in Sec. [III] The main results of the investigations, including time-averaged and instantaneous flow features, are reported in Sec. IV] Finally, conclusions are drawn in Sec. V] with a special reference to flow control applications. 


\section{Numerical approach}

\section{A. Numerical setup}

The direct numerical simulations of the synthetic jet in crossflow are carried out using the open-source CFD code Nek5000 [35]. The present code was deemed to be adequate for the present investigation, since it has been previously employed in several studies concerning continuous jets in crossflow [36], boundary layer suction devices [37], flow around roughness elements [38] and SJs issuing in quiescent environment [39]. This code employs the spectral element method (SEM) to solve the unsteady, incompressible Navier-Stokes equations. The computational domain is partitioned in hexahedral spectral elements: pressure and velocity fields are defined in each element as the combination of the tensor product of 1-dimensional Lagrange polynomials of degree $N$ for the velocity and $N-2$ for the pressure field. For all the simulations shown in the present work it is chosen $N=9$. The time integration, instead, is based on a third-order splitting method. The linear systems arising from the pressure and the velocity discretizations are solved using, respectively, GMRES and conjugate gradient methods, with tolerances of $10^{-7}$ and $10^{-9}$.

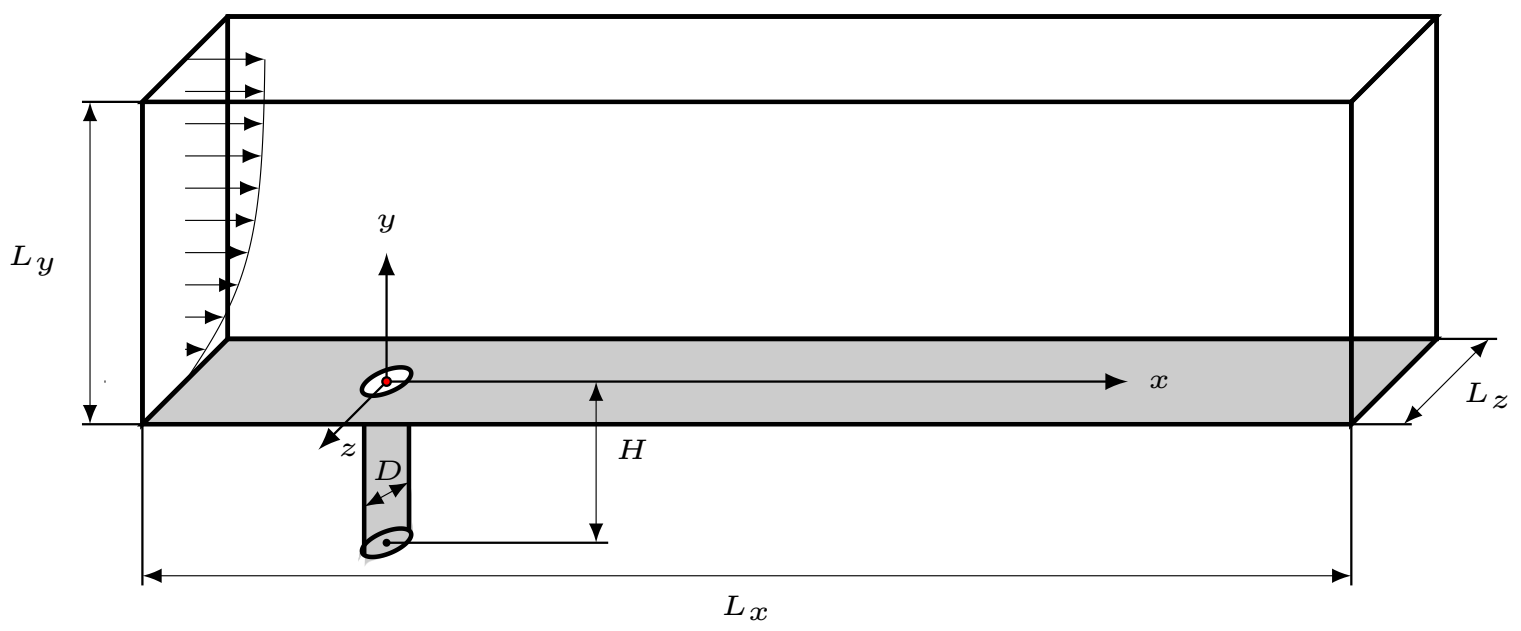

Fig. 1 Computational domain and main geometrical parameters of the simulations.

The numerical domain is shown in Fig. 11: it includes both the external flow field and the jet pipe. All the lengths and the velocities have been made dimensionless against the orifice diameter $D$ and the crossflow velocity $U_{\infty}$. The dimension of the external domain are $L_{x}=55 D, L_{y}=10 D, L_{z}=10 D$, where $x$ denotes the direction of the crossflow, $y$ the direction of the jet and $z$ the spanwise one. The decision of including the inlet pipe in the simulations derives from a recent literature output: in fact, previous studies on continuous jets in crossflow have shown that it is necessary to include the inflow pipe to correctly obtain the flow field at the orifice exit plane [10, 36, 37]. The boundary condition at the crossflow inlet, located $10 D$ upstream of the jet, is a standard Blasius profile, whose displacement thickness is chosen to have a prescribed value of $R e_{\delta_{0}^{*}}$ at the nozzle centerline location (according to the Blasius' similarity solution). The inlet condition for the jet is an oscillating Dirichlet condition: the velocity profile is obtained from Womersley's flow theory, eq. (1):

$$
V(r, t)=\operatorname{Real}\left\{\frac{1}{A}\left[1-\frac{J_{0}\left(i^{3 / 2} W o r\right)}{J_{0}\left(i^{3 / 2} W o\right)}\right] \mathrm{e}^{i \omega t}\right\}, \quad A=V_{a}\left[1-\frac{2}{i^{3 / 2} W o} \frac{J_{1}\left(i^{3 / 2} W o\right)}{J_{0}\left(i^{3 / 2} W o\right)}\right]
$$

where $J_{n}$ is n-th order Bessel function, $r$ the axial distance from the pipe symmetry axis (made dimensionless against the pipe radius), $W o=\sqrt{\frac{\omega D^{2}}{4 v}}$ the Womersley number and $V_{a}$ the velocity amplitude on the symmetry axis. The pipe length is chosen large enough to have a fully-developed pulsating jet flow at its lower end; after a preliminary work $H=5 D$ has been chosen. The present velocity profile (1) has been validated by means of simple axisymmetric computations, which are briefly summarized in Appendix.

No-slip conditions have been applied at the plate and on the nozzle boundary, and periodic boundary conditions are enforced at the spanwise ends $z= \pm L_{z} / 2$. Such a boundary condition is often employed to simulate a synthetic jet array with a reduced computational cost. In this framework $L_{z}$ is the spanwise spacing between the axis of two consecutive 
orifices. This distance comes out as an important project parameter of several active and passive flow control systems, as localized roughness elements, micro-vortex generators and fluidic actuators. Indeed, the control efficiency of an array of actuators is strongly dependent on this distance, being used to modulate the control output in the spanwise direction. The interaction between synthetic jets issuing from orifices in close proximity is widely investigated in literature, both in quiescent conditions and in presence of a crossflow, employing several actuator configurations [40-42]. However, for $L_{z}=10 D$ it is possible to neglect the interaction between adjacent jets, at least in the near-field region.

A natural outflow boundary condition $-p \boldsymbol{n}+\nu \nabla \boldsymbol{U} \cdot \boldsymbol{n}=0$ has been applied at the outlet, while a mixed boundary condition $u=U_{\infty}, v_{y}=w_{y}=0$ is used on the top edge of the boundary layer. The boundary conditions for the pressure field are automatically enforced by the code to ensure the incompressibility constraint of the velocity field.

\section{B. Flow parameters}

The flow field depends on several crossflow and jet non-dimensional parameters: the boundary layer Reynolds number at the nozzle centerline station $R e_{\delta_{0}^{*}}=U_{\infty} \delta_{0}^{*} / v$, the velocity ratio between the jet and the crossflow $R=V_{j} / U_{\infty}$, the reduced frequency $F^{+}=\omega / \omega_{\text {ref }}$, the jet Reynolds number $R e_{j}=V_{j} D / v$ and the pipe dimensionless length $H / D$. In the previous definitions $V_{j}$ is the jet mean velocity amplitude, $\omega=2 \pi f$ its circular frequency, $\omega_{\text {ref }}$ the value of the circular frequency which maximizes the growth rate of the boundary layer instability at the nozzle position, $U_{\infty}$ and $\delta_{0}^{*}$ the boundary layer velocity and thickness at the streamwise position of the pipe axis, $v$ the kinematic viscosity. The influence of $H / D$ on the flow behaviour can be omitted if the flow is fully developed at the pipe inlet, so it will be not investigated in the present study.

The non-dimensional parameters chosen in the simulations are summarized in Table 1 . Two crossflow Reynolds numbers have been selected, $R e_{\delta_{0}^{*}}=500$ and 550, the smaller being slightly below the critical Reynolds number for the Blasius linear instability, whereas the larger one is above this threshold. These flow conditions can be also expressed in terms of local $R e_{x_{0}}=U_{\infty} x_{0} / v$, where $x_{0}$ is the virtual position of the nozzle center, thus obtaining $\operatorname{Re}_{x_{0}} \approx 84500$, and 102000 . Variations in the crossflow Reynolds number have been obtained by changing the (virtual) distance of the jet from the leading edge of the plate. From an experimental point of view, this procedure is equivalent to move the actuator upstream, for a fixed value of the freestream velocity. The velocity ratio is chosen equal to 0.1 , in order to give a small perturbation on the boundary layer and avoid triggering instability mechanisms just downstream the orifice exit. The jet Reynolds number is obtained as a combination of the velocity ratio and the ratio between the boundary layer displacement thickness and the pipe diameter. The latter ratio is chosen as 0.25 for the S2-S4 cases, and 0.227 for the $\mathrm{S} 1$ case. As regards the reduced frequency, the instability frequencies, obtained performing spatial, linear stability analyses at the streamwise location of the nozzle centerline (when the jet is off), for each Reynolds number, are chosen as reference frequencies. The actuation frequency has been chosen equal to the less stable frequency of the boundary layer for the S1 case, and equal to the most unstable frequency for the S3 case, whereas it lies outside the instability range for S2 and S4 simulations. Furthermore, a preliminary simulation (not shown herein), in which the SJ is off (a no-slip condition is applied at the lower end of the pipe) was carried out. The flow field, after a short transient phase, reached a steady state, as expected for the present computational setup. The effect of the inlet pipe was confined in the near field only, consisting in a steady, recirculation region of small extension.

It is worth noting that three different parameters are commonly used to identify the SJ in a quiescent environment, the Reynolds number based on a proper jet average velocity, the Strouhal number and the Stokes number, defined in eq.(2)

$$
R e_{0}=\frac{V_{0} D}{v}, \quad S t_{0}=\frac{\omega D}{V_{0}}, \quad S=\sqrt{\frac{\omega D^{2}}{v}}=2 W o
$$

In the Reynolds number definition $V_{0}$ is the averaged jet velocity, given by eq. (3)

$$
V_{0}=\frac{1}{T} \int_{0}^{T / 2}\left[\int_{A} V(x, y=0, z) d x d z\right] d t
$$

and $T=1 / f$ is the actuation cycle period. The stroke length $L_{0}$ is then defined as $L_{0}=T V_{0}$.

The Stokes number is also the main parameter characterizing the behaviour of the oscillating pipe flow within the pipe and its possible instability and transition to turbulence. The latter parameters are not independent from the aforementioned ones and are summarized in Table 1.

Finally, in several flow control applications the momentum coefficient $C_{\mu}$ is considered in place of the velocity ratio: 
Table 1 Non-dimensional parameters used in the simulations.

\begin{tabular}{ccccc}
\hline Parameter & \multicolumn{4}{c}{ Simulation acronym } \\
\hline S1 & S2 & S3 & S4 \\
\hline Crossflow Reynolds number, $R e_{\delta_{0}^{*}}$ & 500 & 550 & 550 & 550 \\
Crossflow Reynolds number, $R e_{x}$ & $\approx 84500$ & $\approx 100000$ & $\approx 100000$ & $\approx 100000$ \\
Velocity ratio, $R$ & 0.1 & 0.1 & 0.1 & 0.1 \\
BL thickness/jet diameter, $\delta_{0}^{*} / D$ & 0.227 & 0.25 & 0.25 & 0.25 \\
Jet Reynolds Number, $R e_{j}$ & 220 & 220 & 220 & 220 \\
Reduced Frequency, $F^{+}$ & 1 & 0.5 & 1 & 2 \\
Jet Reynolds number, $R e_{0}$ & 70 & 70 & 70 & 70 \\
Strouhal number, $S t$ & 16.8 & 7.41 & 14.8 & 29.2 \\
Stokes number, $S$ & 34.4 & 22.8 & 32.2 & 45.6 \\
Jet momentum ratio, $C_{\mu}$ & 0.01 & 0.01 & 0.01 & 0.01 \\
\hline
\end{tabular}

$$
C_{\mu}=\frac{\rho_{j e t} U_{j e t}^{2}}{\rho_{\infty} V_{\infty}^{2}}
$$

where $\rho_{j e t}$ and $\rho_{\infty}$ are, respectively, the density of the jet and the crossflow. In the present case Eq. (4) simply reduces to $C_{\mu}=R^{2}$.

\section{Results and discussion}

The results of the present investigation will be presented in terms of instantaneous and time-averaged velocity fields, for all the configurations introduced in Table 1 . In particular, simulation S3 is considered as the reference case, and discussed thoroughly in Sec. IV.A whereas the effects of changing the actuation frequency (cases S2 and S4) and boundary layer Reynolds number (case S1) are investigated, respectively, in Sec. IV.B and IV.C.

\section{A. Baseline case $\left(\operatorname{Re}_{\delta_{0}^{*}}=550, \omega=\omega_{r e f}\right)$}

A view of the vortical structures generated by the interaction between the jet and the boundary layer for case $\mathrm{S} 3$ is depicted in Fig. 2, through isosurfaces of the instantaneous $\lambda_{2}$ field [43]. Hairpin-like structures, characterized by a varicose symmetry, are generated at each actuation cycle and advected downstream by the incoming boundary layer flow. The distance between two consecutive vortices in the near field is $\approx 6 D$, and it is close to the wavelength predicted by spatial linear stability for the uncontrolled Blasius proflle at the jet position. The vortices slow down during their motion, and eventually meet each other and merge; the ultimate structure of the flow field in very complex, as vortex interactions, subsequent instabilities and dissipation rise through the domain, however the flow is not fully turbulent at the end of the plate. This feature is confirmed by the fact that the varicose symmetry of the flow is preserved until the end of the domain, which in turn can be related to the short streamwise length of the domain, the low jet-to-BL velocity ratio, and the low Reynolds number of the uncontrolled boundary layer at the jet position.

The effect of the synthetic jet on the boundary layer can be investigated by analyzing the time-averaged controlled flow field. The streamwise velocity field distribution in the symmetry plane is depicted in Fig. 3 (a), whereas the time-averaged vortical structures are represented if Fig. 3 (b). Since the instantaneous flow field is varicose-symmetric with respect to this plane, its maximum deviation from the uncontrolled flow field lies in this plane. The effect of the jet is restricted to a very thin region in the wall normal direction $(0 \leq y / D \leq 2)$, basically consisting in a boundary layer growth enhancement. The time-averaged structures include a horseshoe vortex, located upstream the jet, and two streamwise streaks on the leeward side, similarly to low-Reynolds-number continuous jets in crossflow. The control action of the synthetic jet on the crossflow is better appreciated in Fig. 4 , where the difference $U_{p}=\bar{U}-U_{B}$ between the controlled time-averaged flow field and the corresponding Blasius solution $U_{B}(x, y)$ is depicted. 


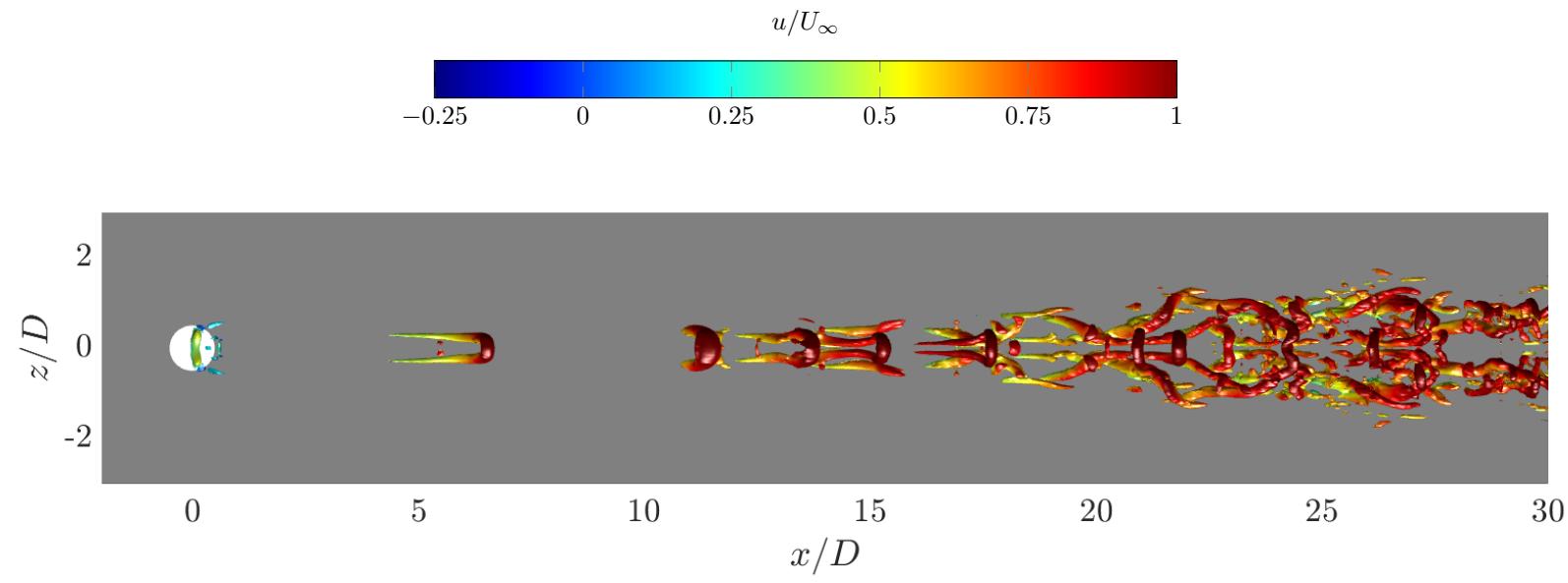

Fig. 2 Instantaneous view of the vortical structures, highlighted by $\lambda_{2}=-0.1$ isosurfaces, and coloured by the instantaneous streamwise velocity. Case $S 3$, tenth cycle, $t / T=1$ (end of suction phase).

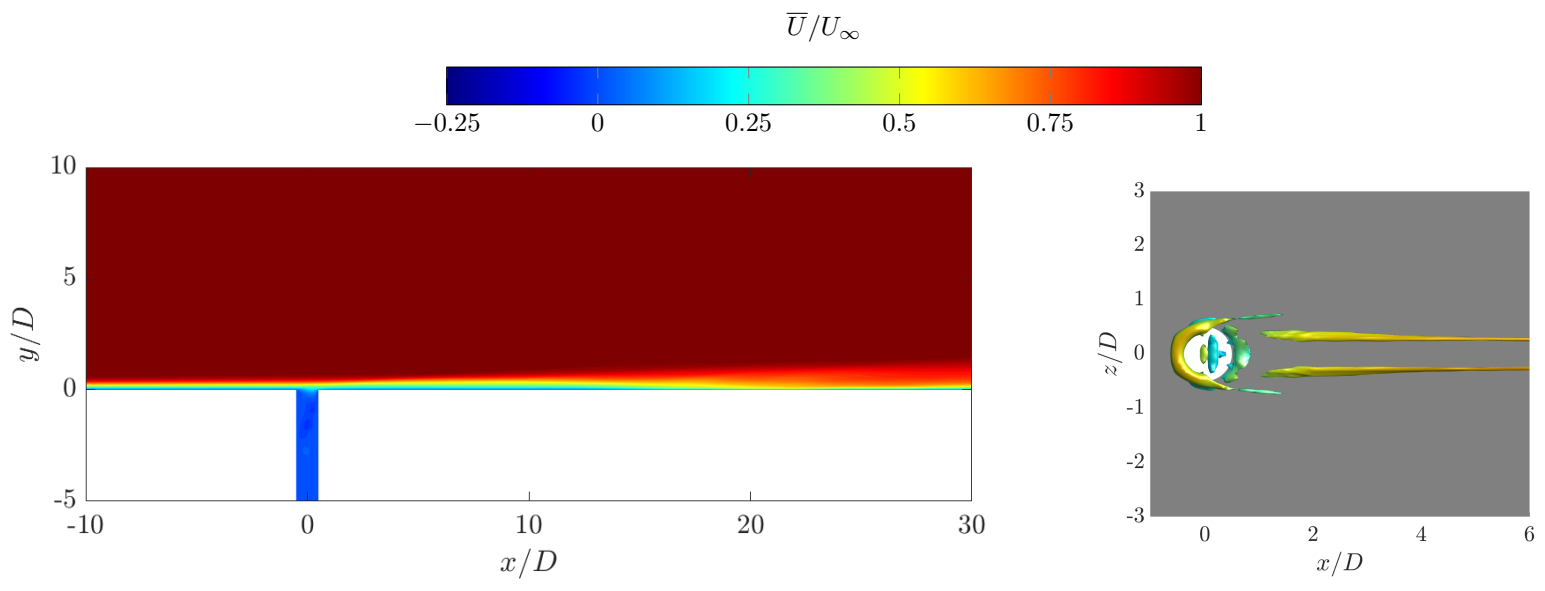

Fig. 3 (left) Time-averaged streamwise velocity field distribution in the symmetry plane for Case S3. (right) Visualization of the time-averaged vortical structures in the near field via $\lambda_{2}=-0.01$ isosurfaces, coloured with streamwise time-averaged velocity.

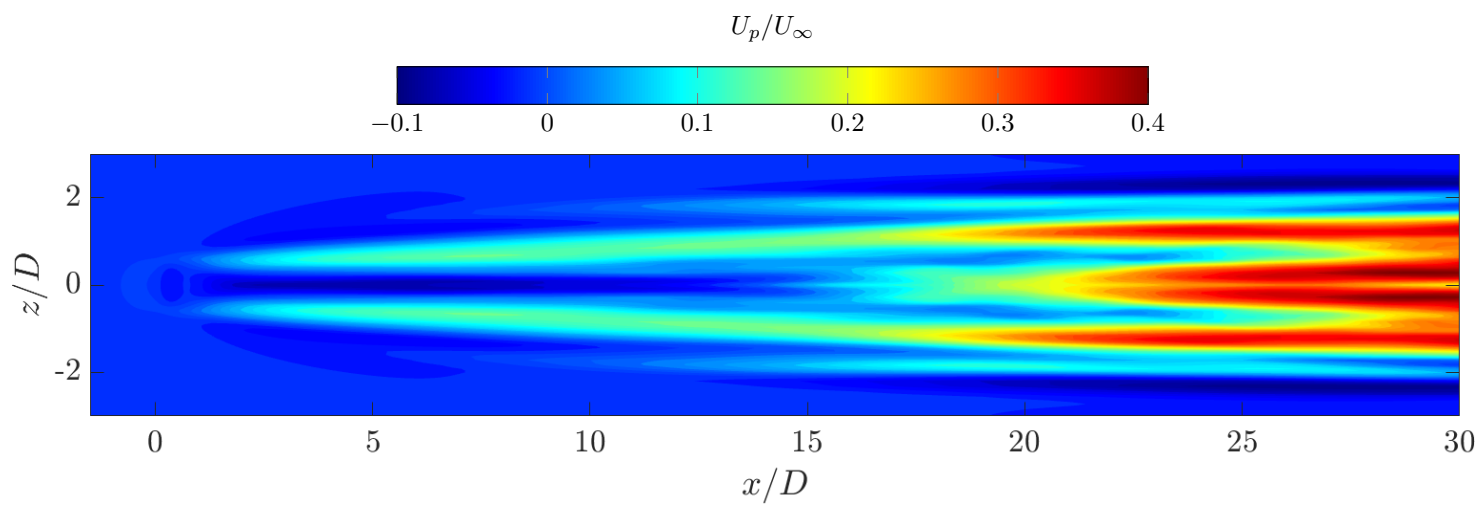

Fig. 4 Mean streamwise deviation of the controlled flow from the Blasius solution; Case S3, $y / \delta_{0}^{*}=0.8$. 
Finally, the time-averaged boundary layer integral parameters, namely the displacement thickness $\bar{\delta}^{*}$, the momentum thickness $\bar{\theta}$ and the shape factor $\bar{H}=\bar{\delta}^{*} / \bar{\theta}$, are shown in Fig. 5. It is possible to distinguish three main regions, while moving downstream from the orifice exit: a transition region, where all the integral parameters grow much faster than a laminar boundary layer. In the following region their growth rate reduces, whereas in the far field the growth rates of the boundary layer parameters increase again, similarly to high-Reynolds-number turbulent boundary layers. It is worth noting that the time-averaged shape factor in the far field is much below the laminar value of 2.6, being comparable with the typical value assumed for turbulent boundary layers of 1.4 [44]. Moreover, also the streamwise distribution of the skin friction coefficient, shown in Fig. 6. collapse onto the turbulent correlation curve $\bar{C}_{f}(x)=0.455 / \log ^{2}\left(0.06 R e_{x}\right)$ [45], after a transition route from their laminar solutions. These facts allow to conclude that a quasi-turbulent state is reached at the end of a domain.

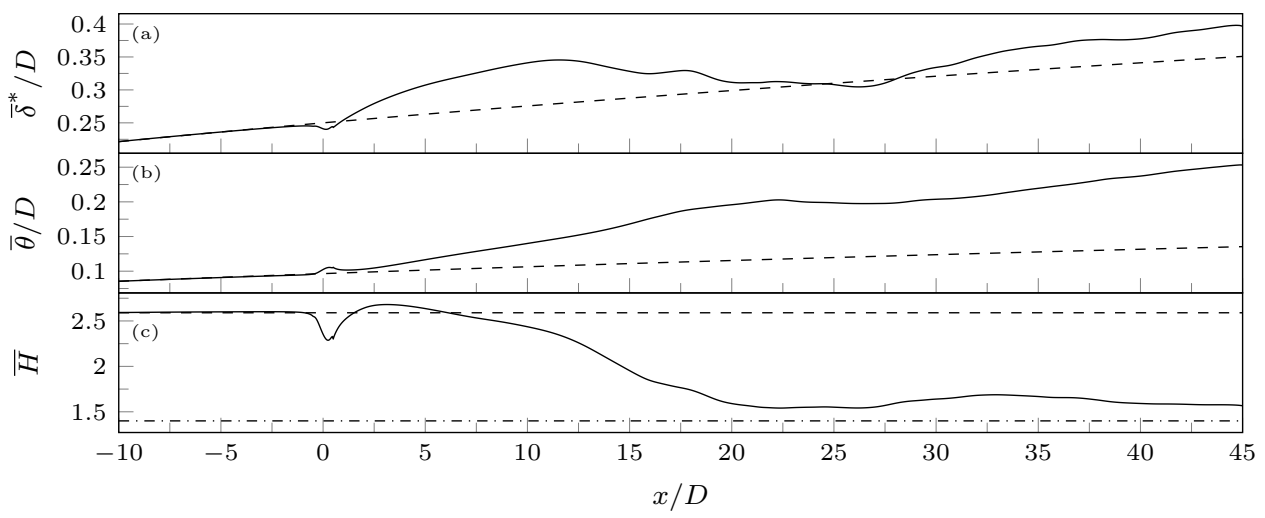

Fig. 5 Streamwise, time-averaged distributions of the boundary layer integral parameters $\bar{\delta}^{*}$ (a), $\bar{\theta}$ (b) and $\bar{H}$ (c) in the symmetry plane for Case S3 (continuous line). The laminar solution is depicted with dashed lines for comparison; in (c) also the value $\bar{H}=1.4$ is shown (dot-dashed line) for reference.

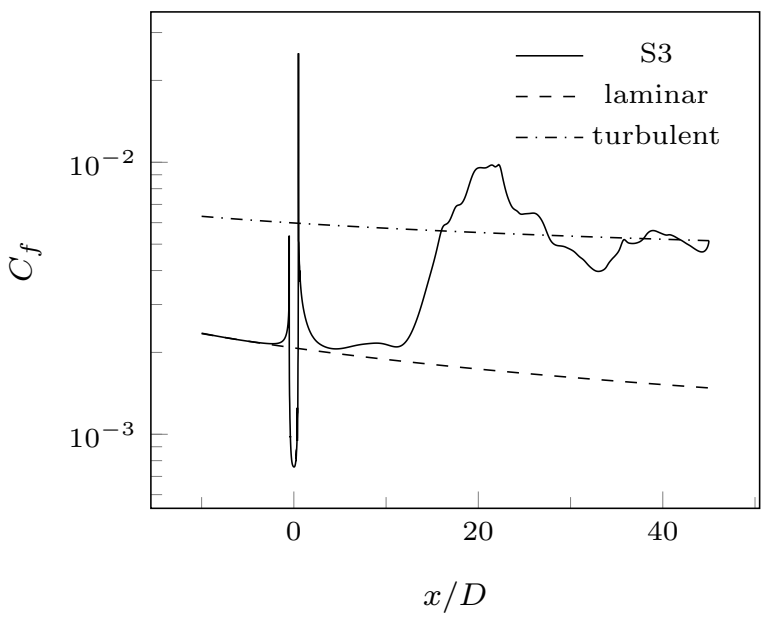

Fig. 6 Time-averaged distribution of the skin friction coefficient $\overline{\mathbf{C}}_{\mathrm{f}}$ along the streamwise direction in the symmetry plane for Case S3. The laminar (Blasius) solution (dashed line) and the turbulent correlation $\overline{\mathbf{C}}_{\mathbf{f}}(\mathbf{x})=\mathbf{0 . 4 5 5} / \log ^{2}\left(0.06 \mathbf{R e}_{\mathbf{x}}\right)$ are shown for comparison.

\section{B. Effect of the actuation frequency}

Fig. 7depicts the evolution of the vortex structures in the streamwise direction for Cases S2 and S4. The topology of the flow field is very similar to Case S3: the vortical structures are still characterized by a varicose symmetry, the vortices spread during their motion and the flow remains laminar till the end of the numerical domain. The main differences 
in the flow development are related to the streamwise and spanwise dimensions of the controlled flow. Firstly, the characteristic streamwise distance of the vortices is reduced for Case S4, owing to the higher actuation frequency; on the contrary, this distance increases for Case S2. Moreover, the spread of the hairpin vortices is still influenced by the actuation frequency: indeed, Case S4 reveals a higher spreading rate the case S3.
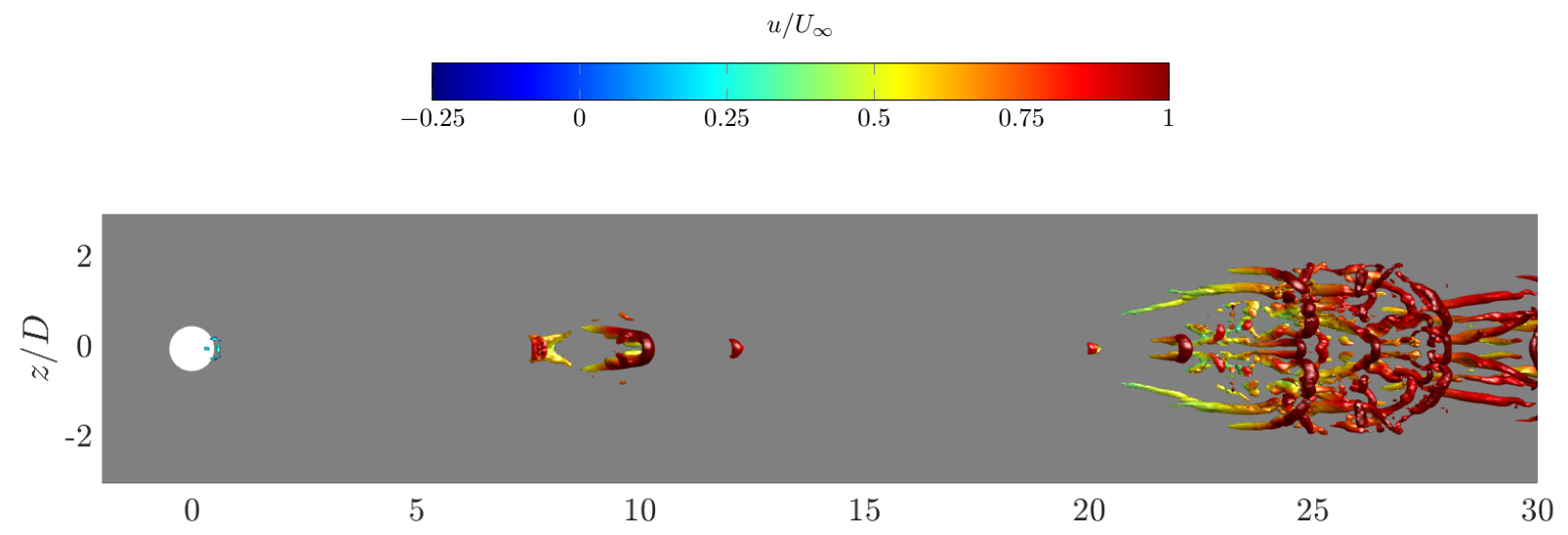

(a)

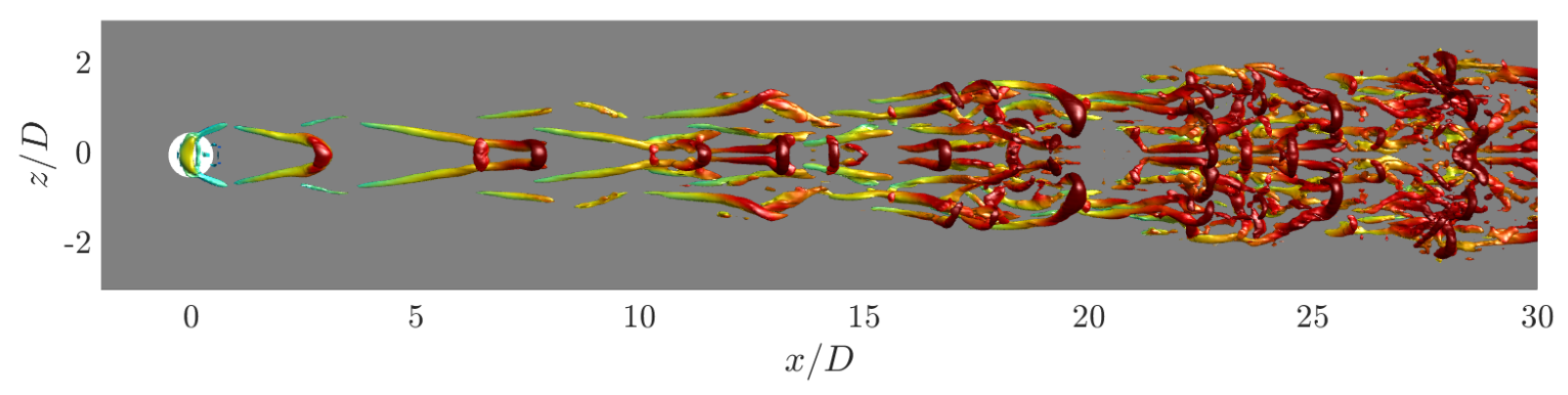

(b)

Fig. 7 Instantaneous view of the vortical structures, highlighted by $\lambda_{2}=-0.1$ isosurfaces, and coloured by the instantaneous streamwise velocity. (a) Case $S 2$, (b) case $S 4$, tenth cycle, $t / T=1$ (end of suction phase).

Time-averaged results, again, can be used to assess the capability of the SJ actuation to modify the boundary layer development. Fig. 8 shows the streamwise distribution of the boundary layer parameters: the strongest deviation from the laminar solution towards a quasi-turbulent flow is observed for the S4 case, which rapidly departs from the laminar solution, as shown by the shape factor diagram. Similar conclusions can be drawn by analyzing the time-averaged distribution of the skin friction coefficient (Fig. 9).

\section{Effect of the Reynolds number}

Fig. 10. depicts the evolution of the vortical structures at the end of the tenth actuation cycle for Cases S1 and S3. It can been seen that reducing the boundary layer Reynolds number below its critical value does not modify the behaviour of the flow. Indeed, the vortical structures are still advected dowstream by the crossflow, leading again to a complicated, transitional flow (as shown by the $C_{f}$ streamwise distribution in Fig. 11). However, the spacing between the vortices, the initial vortex celerity and their spreading rate are slightly different from the baseline case. In fact, these differences can be attributed to two conflicting causes: it must be kept in mind that the $\mathrm{S} 1$ case is obtained for $F^{+}=1$, but the frequency which maximizes the TS growth rate is decreasing with the crossflow Reynolds number. For this reason, S1 case is characterized by a smaller $\delta_{0}^{*}$ and a higher applied frequency $F$. These parameters act in opposite ways on the transition process, yielding to similar $C_{f}$ distributions for the considered cases. 


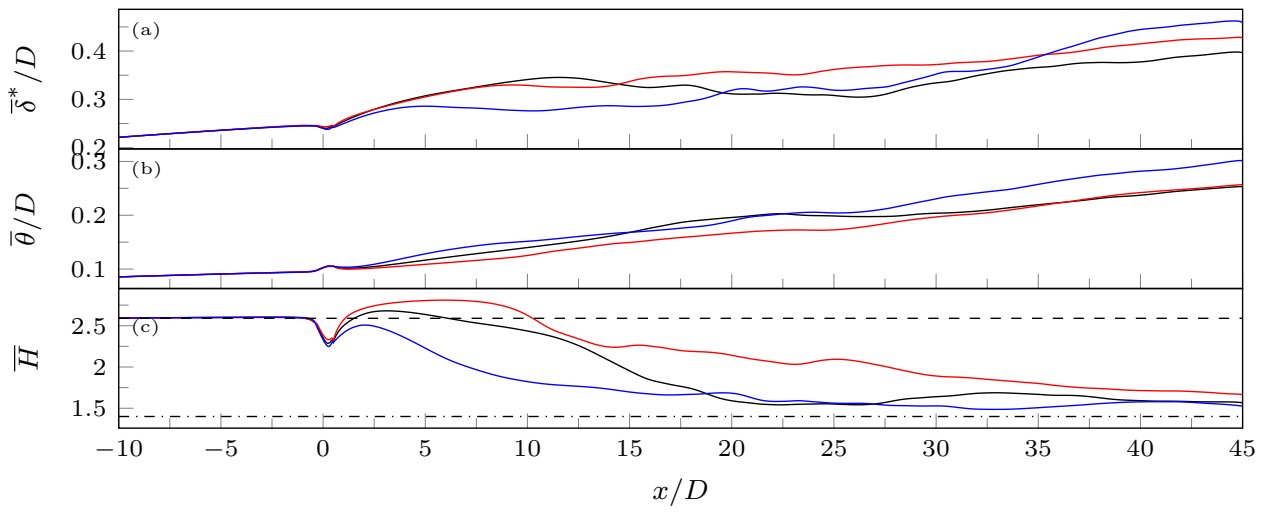

Fig. 8 Streamwise, time-averaged distribution of the boundary layer integral parameters $\bar{\delta}^{*}, \bar{\theta}$ and $\bar{H}$ in the symmetry plane for Case S2 (red line), S3 (black), S4 (blue). The laminar solution is depicted with dashed lines for comparison; in (c) also the value $\bar{H}=1.4$ is shown (dot-dashed line) for reference.

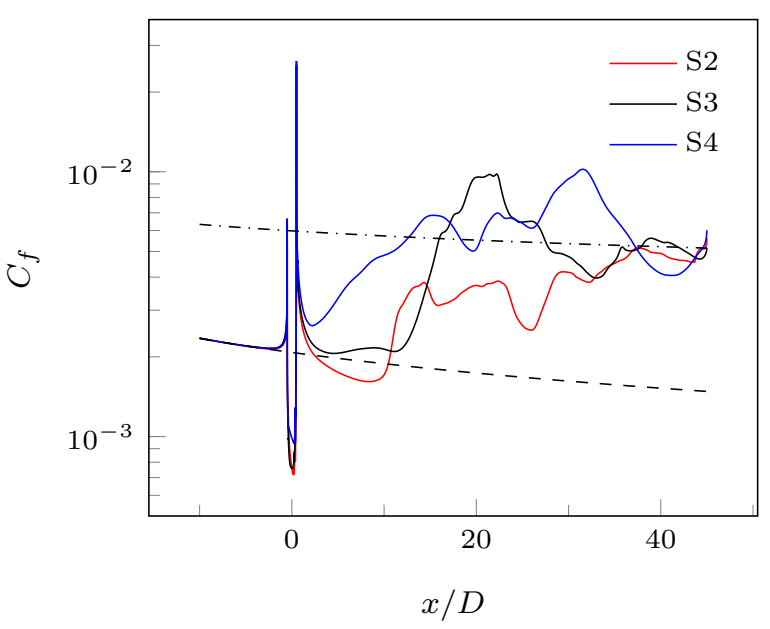

Fig. 9 Time-averaged distribution of the skin friction coefficient $\overline{\mathbf{C}}_{\mathrm{f}}$ along the streamwise direction in the symmetry plane for Case S2 (red line), S3 (black), S4 (blue). The laminar (Blasius) solution (dashed lines) and the turbulent correlation $\bar{C}_{\mathbf{f}}(\mathbf{x})=0.455 / \log ^{2}\left(0.06 R e_{x}\right)$ are shown for comparison. 


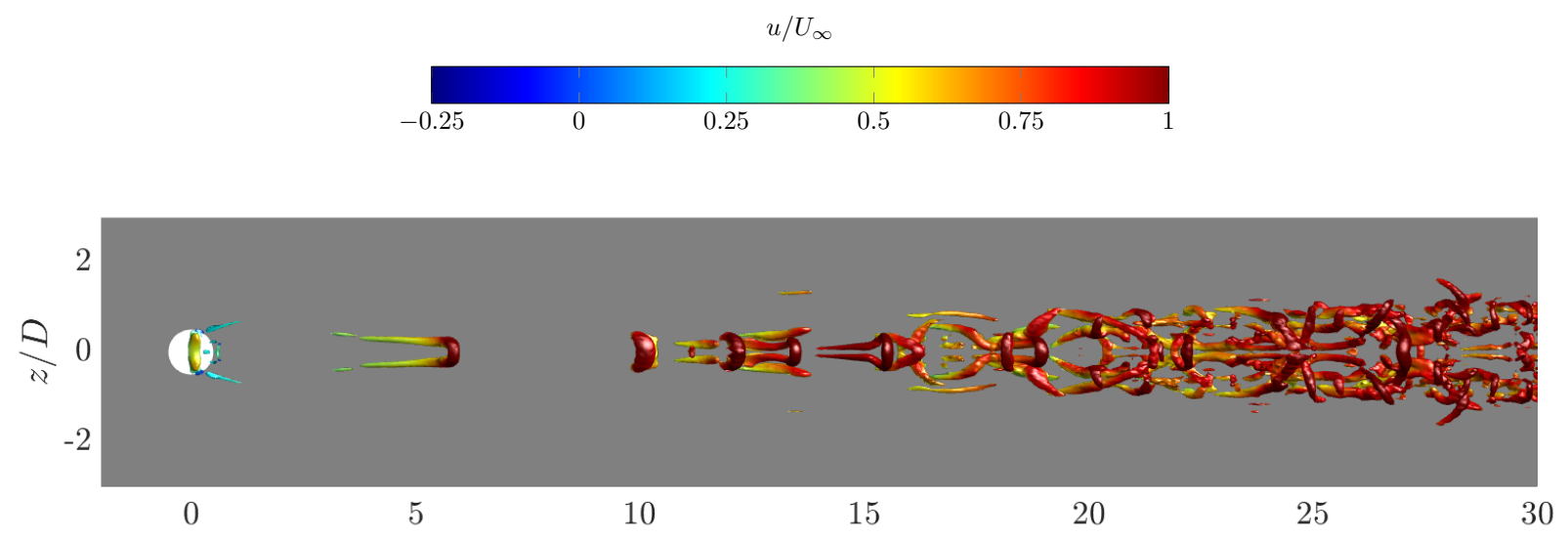

(a)

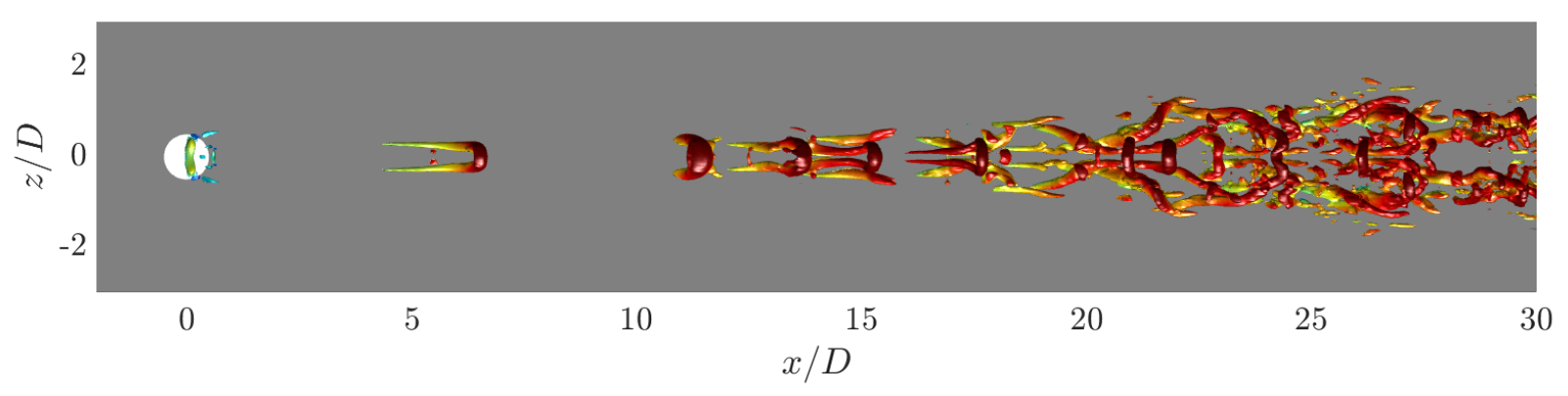

(b)

Fig. 10 Instantaneous view of the vortical structures, highlighted by $\lambda_{2}=-0.1$ isosurfaces, and coloured by the instantaneous streamwise velocity. (a) Case $S 1$, (b) case $S 3$, tenth cycle, $t / T=1$ (end of suction phase).

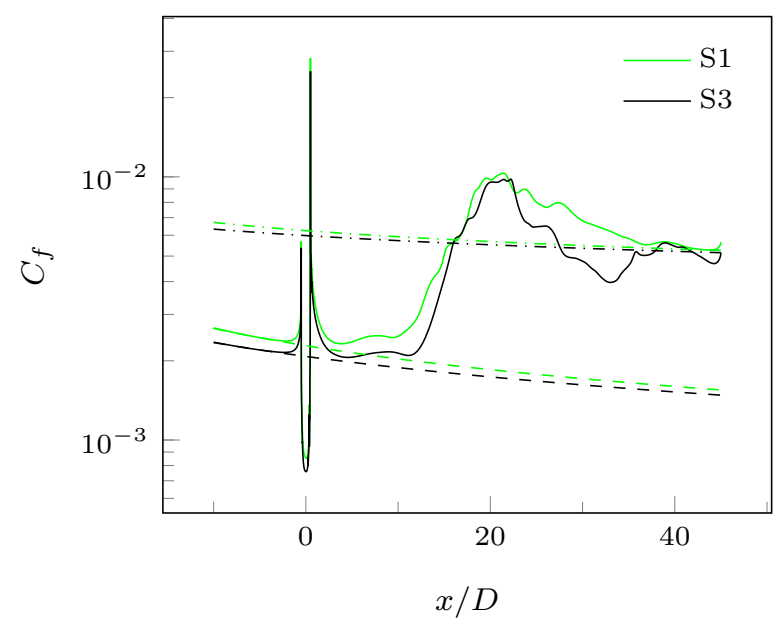

Fig. 11 Time-averaged distribution of the skin friction coefficient $\bar{C}_{f}$ along the streamwise direction in the symmetry plane for Case S1 (green line) and S3 (black). The laminar (Blasius) solutions (dashed lines) and the turbulent correlation $\bar{C}_{\mathrm{f}}(\mathbf{x})=0.455 / \log ^{2}\left(0.06 \mathrm{Re}_{\mathrm{x}}\right)$ are shown for comparison. 


\section{Discussion}

The flow field topology generated by the jet-boundary layer interaction perfectly resembles the unsteady flow field generated by an isolated cylindrical roughness immersed in a boundary layer flow. The reader can compare, for instance, Fig. 2] of the present work with the numerical campaign carried out in [38] for similar crossflow conditions. It is well known nowadays that roughness elements are able to produce streamwise streaks, which, if stable, attenuate the growth of the Tollmien-Schlichting waves and, as a consequence, delay the transition to turbulence. Nonetheless, in many practical situations the streaks generated by the cylindrical element can be unstable themselves, leading to self-sustained hairpin structures and, as a consequence, early transition to turbulence. This result is also well known in literature [46], indeed roughness elements are often used as passive flow control devices to promote transition.

From a flow control perspective, however, the performances of such devices drop in off-design conditions, as they work properly only within a small range of boundary layer Reynolds numbers. Synthetic jet actuators, on the contrary, assure good performances in a wider range of freestream conditions, owing to their flexibility and wide applied frequency interval. In particular, the actuation frequency and applied voltages can be changed during their operation in order to handle different crossflow conditions.

\section{Conclusions and future work}

This paper deals with direct numerical simulations of the flow field generated by the interaction between a synthetic jet (produced by a zero-net-mass-flux device) and a boundary layer crossflow, for the purpose of recognizing the vortical structures arising in the flow field. Differently from previous virtual aeroshaping studies, the original aspect of this work consists in the fact that the actuation frequency is next to the characteristic frequency of the crossflow, namely it is equal to the unstable (or less stable) frequency, so as to deepen the understanding of how the synthetic jet modifies the modes of the uncontrolled flow. A baseline flow configuration has been initially chosen, and additional simulations have been carried out in order to ascertain the effects of the boundary layer Reynolds number and applied frequency on the controlled flow fields. The instantaneous controlled flow field is dominated by hairpin structures for all the considered numerical setups, characterized by a varicose symmetry, similarly to the unsteady flow behind a cylindrical roughness. The time-averaged flow field, instead, is characterized by streaky structures, generated at the spanwise ends of the orifice, and a horseshoe vortex, located upstream of the jet. The analysis of the boundary layer integral parameters revealed that the overall effect of the jet is to promote transition, since the time-averaged shape factor and the skin friction coefficient values are in close agreement with turbulent correlation ones. Moreover, the transition region is smaller if higher applied frequencies are chosen.

As a final comment, it is worth noting that the present study approaches the problem of realizing a reduced-order modeling of a piezo-driven synthetic jet in crossflow to suppress separated flows, based on simulations and lumped element modeling of the actuator. In particular, the analysis of the boundary layer shape factor $\bar{H}$ shows that SJ actuators is capable of making flows more resistant to separation. Moreover, their application could be preferred to passive flow control devices, as roughness elements or micro-vortex generators (MVG), since changes in the BL characteristics can be tackled by changing the actuation frequency and the applied voltage on-the-fly.

\section{Appendix}

\section{A. Validation of the inlet profile}

Preliminary simulations of the oscillating pipe flow have been carried out to validate the analytical velocity profile used in the present work. Two-dimensional (axysimmetric) simulations of the pipe in cylindrical coordinates have been performed, enforcing a top-hat, oscillating velocity profile at the pipe inlet. The velocity profiles, after a short development region, converge to the analytical solution of Eq. 11, as shown in Fig. 12, where $D$ is the pipe diameter, and $r$ the radial coordinate; open circles represent the numerical data, and the continuous lines the analytical solution. Both the Stokes layer (of order $D / W o$ ) and the central region are well modelled by the analytic solution.

\section{B. Choice of the actuation frequency via Linear Stability Theory}

The actuation frequency used in the simulation have been obtained performing a spatial, linear stability analysis of the unactuated boundary layer at the streamwise position of the centerline of the nozzle. In this framework the velocity field $\boldsymbol{u}^{\prime}$ and the pressure $p^{\prime}$ perturbation fields are decomposed using the ansatz in Eq. (5) 


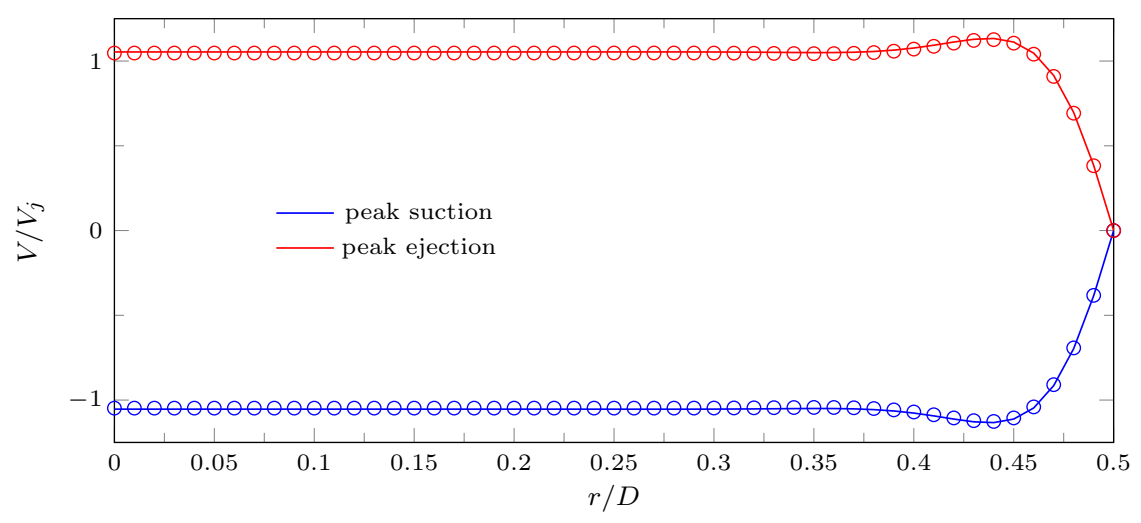

Fig. 12 Comparison between analytical (continuous line) and numerical (open circles) results of the oscillating pipe flow. $\operatorname{Re}_{\mathbf{j}}=220$, Wo $=26$.

$$
\left\{\begin{array}{c}
u^{\prime}(x, y, z, t) \\
v^{\prime}(x, y, z, t) \\
w^{\prime}(x, y, z, t) \\
p^{\prime}(x, y, z, t)
\end{array}\right\}=\left\{\begin{array}{c}
\hat{u}(y) \\
\hat{v}(y) \\
\hat{w}(y) \\
\hat{p}(y)
\end{array}\right\} e^{i \alpha x} e^{i \beta z} e^{-i \omega^{*} t}+c . c .
$$

which is introduced in the linearized Navier-Stokes equations. In the present work the spatial stability analysis for the Blasius boundary layer has been carried out by means of a Chebyschev collocation method; the results, in terms of marginal stability curve $\left(\alpha_{i}=0\right)$ and spatial growth rate $-\alpha_{i}$ at four different Reynolds numbers are shown in Fig. 13 The nondimensional frequency $F=\omega^{*} / R e_{\delta_{0}^{*}} \cdot 10^{6}$ has been introduced to scale the frequency along the streamwise direction and eliminating the dependence of the displacement thickness on the Reynolds number. As is known, this allows to have a constant frequency for various downstream positions [47].
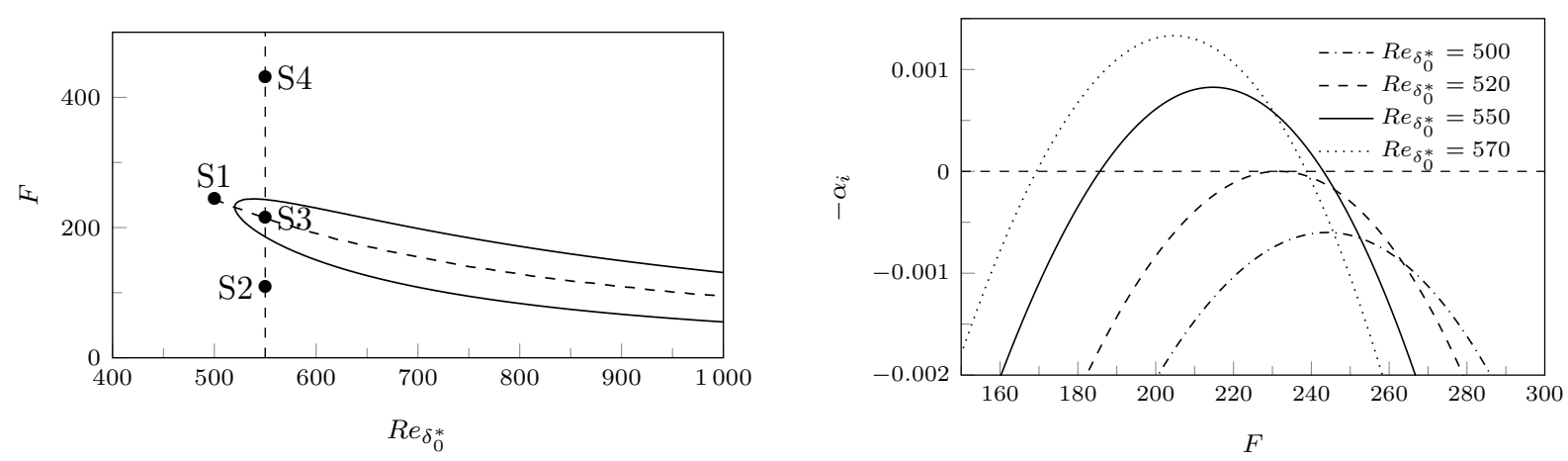

Fig. 13 (left) Marginal stability curve for the spatial stability of the Blasius boundary layer. The dashed line connects the frequencies of maximum growth rate for each Reynolds number. Filled circles are used to show the computational setups of S1-S4 simulations; (right) Spatial growth rate for different Reynolds numbers.

The $\omega_{\text {ref }}$ values to be used in the simulations are obtained as follows. For each Reynolds number $R e_{\delta_{0}^{*}}$ the value of $\omega^{*}$ which maximizes the spatial growth (namely, that of the most amplified wave) is first evaluated; this frequency is then made dimensional by multiplying it by the reference quantity $U_{\infty} / \delta_{0}^{*}$.

\section{Acknowledgments}

Andrea Palumbo was supported by Università degli Studi di Napoli Federico II and Compagnia di San Paolo under the STAR Program 2018. Part of this work was supported by a grant of HPC time on CINES supercomputer Occigen under the project A0052A062 and on CINECA supercomputer MARCONI under the ISCRA-C project INJECT. 


\section{References}

[1] Glezer, A., and Amitay, M., "Synthetic jets," Annual review of fluid mechanics, Vol. 34, No. 1, 2002, pp. 503-529.

[2] Zong, H., Chiatto, M., Kotsonis, M., and de Luca, L., "Plasma synthetic jet actuators for active flow control," Actuators, Vol. 7 , Multidisciplinary Digital Publishing Institute, 2018, p. 77.

[3] Arena, M., Chiatto, M., Amoroso, F., Pecora, R., and de Luca, L., "Feasibility studies for the installation of Plasma Synthetic Jet Actuators on the skin of a morphing wing flap," Active and Passive Smart Structures and Integrated Systems XII, Vol. 10595, International Society for Optics and Photonics, 2018, p. 105950M.

[4] Jansen, K. E., Rasquin, M., Farnsworth, J. A., Rathay, N., Monastero, M. C., and Amitay, M., "Interaction of a Synthetic Jet with Separated Flow over a Vertical Tail,” AIAA Journal, Vol. 56, No. 7, 2018, pp. 2653-2668.

[5] Crowther, W. J., and Gomes, L., "An evaluation of the mass and power scaling of synthetic jet actuator flow control technology for civil transport aircraft applications," Proceedings of Institution of Mechanical Engineer Part I: Systems and Control Engineering, Vol. 222, 2008, pp. 357-372. doi:10.1243/09596518JSCE519.

[6] Ma, L.-Q., and Feng, L.-H., "Vortex formation and evolution for flow over a circular cylinder excited by symmetric synthetic jets," Experimental Thermal and Fluid Science, Vol. 104, 2019, pp. 89-104.

[7] Smith, B., and Glezer, A., "Jet vectoring using synthetic jets," J. Fluid Mech., Vol. 458, 2002, pp. 1-34. doi:10.1017/ S0022112001007406.

[8] Tamburello, D. A., and Amitay, M., "Active control of a free jet using a synthetic jet," International journal of heat and fluid flow, Vol. 29, No. 4, 2008, pp. 967-984.

[9] Regan, M. A., and Mahesh, K., "Global linear stability analysis of jets in cross-flow," J. Fluid Mech., Vol. 828, 2017, pp. 812-836.

[10] Iyer, P., and Mahesh, K., "A numerical study of shear layer characteristics of low-speed transverse jets," J. Fluid Mech., Vol. 790, 2016, pp. 275-307.

[11] Kelso, R., Lim, T., and Perry, A., “An experimental study of round jets in cross-flow,” J. Fluid Mech., Vol. 306, 1996, pp. $111-144$.

[12] Megerian, S., Davitian, J., de B. Alves, L., and Karagozian, A., “Transverse-jet shear-layer instabilities. Part 1. Experimental studies," J. Fluid Mech., Vol. 593, 2007, pp. 93-129.

[13] Jabbal, M., and Zhong, S., "The near wall effect of synthetic jets in a boundary layer," International Journal of Heat and Fluid Flow, Vol. 29, No. 1, 2008, pp. 119-130.

[14] Zhou, J., and Zhong, S., "Coherent structures produced by the interaction between synthetic jets and a laminar boundary layer and their surface shear stress patterns," Computers \& Fluids, Vol. 39, No. 8, 2010, pp. 1296-1313.

[15] Xia, X., and Mohseni, K., "Flow characterization and modeling of strong round synthetic jets in crossflow," AIAA Journal, Vol. 55, No. 2, 2016, pp. 389-402.

[16] Milanovic, I. M., and Zaman, K., "Synthetic jets in cross-flow," AIAA journal, Vol. 43, No. 5, 2005, pp. 929-940.

[17] Schaeffler, N., "The interaction of a synthetic jet and a turbulent boundary layer," 41st Aerospace Sciences Meeting and Exhibit, 2003, p. 643.

[18] Wu, D. K., and Leschziner, M. A., "Large-eddy simulations of circular synthetic jets in quiescent surroundings and in turbulent cross-flow," International Journal of Heat and Fluid Flow, Vol. 30, No. 3, 2009, pp. 421-434.

[19] Berk, T., Hutchins, N., Marusic, I., and Ganapathisubramani, B., "Trajectory of a synthetic jet issuing into high-Reynolds-number turbulent boundary layers," Journal of Fluid Mechanics, Vol. 856, 2018, pp. 531-551.

[20] Berk, T., and Ganapathisubramani, B., "Effects of vortex-induced velocity on the development of a synthetic jet issuing into a turbulent boundary layer," Journal of Fluid Mechanics, Vol. 870, 2019, pp. 651-679.

[21] Zong, H., and Kotsonis, M., "Effect of velocity ratio on the interaction between plasma synthetic jets and turbulent cross-flow," Journal of Fluid Mechanics, Vol. 865, 2019, pp. 928-962. 
[22] Mittal, R., and Rampunggoon, P., "On the virtual aeroshaping effect of synthetic jets," Physics of Fluids, Vol. 14, No. 4, 2002, pp. 1533-1536.

[23] Chatlynne, E., Rumigny, N., Amitay, M., and Glezer, A., "Virtual aero-shaping of a Clark-Y airfoil using synthetic jet actuators," 39th Aerospace Sciences Meeting and Exhibit, 2001, p. 732.

[24] Dandois, J., Garnier, E., and Sagaut, P., "Numerical simulation of active separation control by a synthetic jet," Journal of Fluid Mechanics, Vol. 574, 2007, pp. 25-58.

[25] Rowley, C. W., and Dawson, S. T., "Model reduction for flow analysis and control," Annual Review of Fluid Mechanics, Vol. 49, 2017, pp. 387-417.

[26] Gallas, Q., Holman, R., Nishida, T., Carroll, B., Sheplak, M., and Cattafesta, L., "Lumped Element Modeling of PiezoelectricDriven Synthetic Jet Actuators,” AIAA Journal, Vol. 41, 2003, pp. 240-247. doi:10.2514/2.1936.

[27] Chiatto, M., Capuano, F., Coppola, G., and de Luca, L., "LEM characterization of synthetic jet actuators driven by piezoelectric element: A review," Sensors (Switzerland), Vol. 17, No. 6, 2017, p. 1216. doi:10.3390/s17061216.

[28] Zong, H., Wu, Y., Li, Y., Song, H., Zhang, Z., and Jia, M., "Analytic model and frequency characteristics of plasma synthetic jet actuator," Physics of Fluids, Vol. 27, 2015, pp. 1-21. doi:10.1063/1.4908071.

[29] Chiatto, M., Palumbo, A., and de Luca, L., "A Calibrated Lumped Element Model for the Prediction of PSJ Actuator Efficiency Performance," Actuators, Vol. 7, Multidisciplinary Digital Publishing Institute, 2018, p. 10.

[30] Chiatto, M., Capuano, F., and de Luca, L., "Numerical and experimental characterization of a double-orifice synthetic jet actuator," Meccanica, Vol. 53, No. 11-12, 2018, pp. 2883-2896. doi:10.1007/s11012-018-0866-7.

[31] Chiatto, M., Palumbo, A., and de Luca, L., "Design approach to predict synthetic jet formation and resonance amplifications," Experimental Thermal and Fluid Science, Vol. 107, 2019, pp. 79-87.

[32] Lindstrom, A., and Amitay, M., "Effect of Orifice Geometry on Synthetic Jet Evolution,” AIAA Journal, 2019 , pp. 1-12.

[33] Shi, X.-D., Feng, L.-H., and Wang, J.-J., "Evolution of elliptic synthetic jets at low Reynolds number," Journal of Fluid Mechanics, Vol. 868, 2019, pp. 66-96.

[34] Palumbo, A., Chiatto, M., and de Luca, L., "Measurements versus Numerical Simulations for Slotted Synthetic Jet Actuator," Actuators, Vol. 7, Multidisciplinary Digital Publishing Institute, 2018, p. 59.

[35] Fischer, P. F., Lottes, J. W., and Kerkemeier, S. G., “nek5000 Web page,”, 2008.

[36] Peplinski, A., Schlatter, P., and Henningson, D. S., "Global stability and optimal perturbation for a jet in cross-flow," European Journal of Mechanics-B/Fluids, Vol. 49, 2015, pp. 438-447.

[37] Brynjell-Rahkola, M., Hanifi, A., and Henningson, D. S., "On the stability of a Blasius boundary layer subject to localised suction,” Journal of Fluid Mechanics, Vol. 871, 2019, pp. 717-741.

[38] Bucci, M., Puckert, D., Andriano, C., Loiseau, J.-C., Cherubini, S., Robinet, J.-C., and Rist, U., "Roughness-induced transition by quasi-resonance of a varicose global mode," Journal of Fluid Mechanics, Vol. 836, 2018, pp. 167-191.

[39] Capuano, F., Palumbo, A., and de Luca, L., "Comparative study of spectral-element and finite-volume solvers for direct numerical simulation of synthetic jets,” Computers \& Fluids, Vol. 179, 2019, pp. 228-237.

[40] Palumbo, A., Della Pia, A., Chiatto, M., and de Luca, L., "Numerical study on the flow field generated by a double-orifice synthetic jet device," Proceedings of the 24rd Conference of the Italian Association of Theoretical and Applied Mechanics, 2019.

[41] Watson, M., Jaworski, A. J., and Wood, N. J., "Contribution to the understanding of flow interactions between multiple synthetic jets," AIAA journal, Vol. 41, No. 4, 2003, pp. 747-749.

[42] McGlynn, E., Tran, S., and Sahni, O., "Large Eddy Simulation of Flow Interactions of Segmented Synthetic Jets on an Airfoil," 47th AIAA Fluid Dynamics Conference, 2017, p. 3310.

[43] Jeong, J., and Hussain, F., “On the identification of a vortex,” Journal of Fluid Mechanics, Vol. 285, 1995, p. 69-94.

[44] Schlichting, H., and Gersten, K., Boundary-layer theory, Springer, 2016. 
[45] White, F. M., Viscous fluid flow, Vol. 3, McGraw-Hill New York, 2006.

[46] Klebanoff, P. S., and Tidstrom, K. D., "Mechanism by Which a Two-Dimensional Roughness Element Induces Boundary-Layer Transition," Physics of Fluids, Vol. 15, No. 7, 1972, pp. 1173-1188.

[47] Schmid, P. J., and Henningson, D. S., Stability and transition in shear flows, Vol. 142, Springer Science \& Business Media, 2012. 